\title{
Adaptive Algorithm Design for Cooperative Hunting in Multi-Robots
}

\author{
Poorva Agrawal \\ Symbiosis Institute of Technology, Symbiosis International (Deemed University) Maharashtra, Pune 412115, India \\ E-mail: poorva.agrawal@sitpune.edu.in \\ Himanshu Agrawal \\ Symbiosis Institute of Technology, Symbiosis International (Deemed University) Maharashtra, Pune 412115, India \\ E-mail: himanshu.agrawal@sitpune.edu.in
}

Received: 02 March 2018; Accepted: 20 May 2018; Published: 08 December 2018

\begin{abstract}
The multi-robot cooperative planning is gained significant attention in recent past mainly for the evaders hunting task. In evaders hunting, the robot nodes required to recognize their other team members and considering their current positions and capabilities to catch the stationary or moving evaders effectively through the cooperating path planning approach. The primary challenge to design cooperative multi-robot evader hunting system is efficient and adaptive coordination of multiple autonomous mobile robots with less delay and communication overhead in presence of big-size obstacles. The current solutions suffered from repeated hunting problem under the inaccessible network conditions due to the presence of big-size obstacles and ineffective utilization of known nodes information. In this paper, to alleviate the problem of repeated hunting and inefficient catching of all evaders in the network, we proposed the adaptive Bio-inspired Neural Network (ABNN) using the new shunting equation with the capability of adaptive hunting of all evaders in the system. We design $A B N N$ based on the implicit robot to predict the next path to catch evaders efficiently by real robots. The use of implicit robot helps to prevent the big sized evaders and efficiently utilize the evader's information. The simulation results demonstrate that $\mathrm{ABNN}$ performs efficient evaders hunting under the presence of big size obstacles.
\end{abstract}

Index Terms-Multi-robots, bio-inspired neural network, evaders, cooperative hunting problem and implicit robot.

\section{INTRODUCTION}

The path planning problem using the single robot extensively studied during 1990's [1]. However, the path planning tasks using multi-robot is more complex and challenging research problem especially the case where networks consist of a large number of robots and more dynamics. The robotics systems are composed of both stationary robots and mobile robots. The multi-robot cooperation with goal path planning, avoiding obstacles, catching the evaders is one of the challenging research problems. The conventional strategy to solve the problem of multi-robot co-operation was the time dimension added to the robot configuration space [2]. The multirobot cooperation was performed using either of two approaches such as centralized techniques and distributed techniques. The centralized methods always try to deliver complete solution; however, such practices need more time and computational overhead due to the severe complexity of the particular problem. In recent past, the probabilistic roadmaps based methods alleviate this problem [3]. On the other hand, the distributed techniques minimize the complexity through the autonomous path planning for every robot in the network. Then the individual paths can be merged using different techniques such as path coordination methods [4], potential fields [5], or priorities assignment methods [6]. In all network conditions, such distributed methods fail to deliver the complete solution and hence may not produce the optimal solution. Therefore, it is required to have more dynamic and adaptive techniques for multi-robot based cooperative path planning through the coordination and merging of paths [7-8]. The path planning methods based on wireless routing reported in [32]-[34] for underwater wireless networks.

Designing the cooperative strategies is essential to research challenges in different real-time applications of multi-robot systems. The system with a large number of robots needs the extra efforts for the multi-robots communication. Many cooperation methods mainly rely on the explicit negotiations among the robots, and hence this will require the substantial amount of transmission [9]. Such problem becomes even more complicated when all the communications among the robots should synchronize in such a way that dynamic changes in system environment do not lead to miss communications before they get completed. Therefore, it is vital to minimize the communication steps required among the robots; else the dynamic real-time planning will not be applicable [10-12].

Over the years, things have been changed and improved slightly. The coordination in multi-robots systems gained the significant attention, as using the team 
of robots tasks are completed efficiently and fast. However, still, there are some problems faced by multirobots coordination systems such as dynamic positioning of a robot, imperfect communication systems, inefficient robots cooperation, etc [11]. Over the past decade, there is the number of research studies reported on multi-robot cooperation systems. However, the main challenges with such methods are target localization, allocation of tasks, obstacle and collisions prevention, reducing the pursuing and finding steps, etc. [13-14]. The multi-robot hunting is even more challenging and complex problem in multirobot cooperation systems as it includes all such subproblems. There is the number of solutions presented to solve the problem of multi-robot based cooperative hunting [15-17]. Such conventional methods classified into two categories such as location-based and sensorbased methods. In location-based ways, the target location is known in advance in which the artificial intelligence based cooperative techniques are used to hunt the targets [18]. Whereas in sensor-based methods, traditional control theory is adopted by most of the researchers to perform the task of target hunting in the unknown environment using incoming sensor information [19]. But both approaches suffered from the limitations; such as sensor-based methods are not scalable as they only depend on sensor data to perform the task of cooperative hunting, whereas the location-based techniques solely depend on location information of targets which is practically not possible under real-time environments, where the positions are frequently changing.

The problems of such methods solved by a method reported in [20]. The Jianjun Ni et al. [20] introduced the dynamic BNN method for the real-time cooperative hunting by multi-robots by considering the evaders positions as an unknown and infrequently changing environment, i.e., the plan designed to handle the network dynamics. The author further extended BNN based work with different conditions and situations. The number of works reported in [21-28] to solve the multi-robot based hunting problem in under different network environment recently. However, the methods mainly suffered from problems like such methods failed to address the cases when there are obstacles with bigger size than sensors detection range and hence this leads to repeated hunting problem and this method fails to utilize evader information efficiently and therefore this leads to the extra communication overhead and delay.

In this paper, we are working on the problem of cooperative hunting of many evaders by the multiple robots in dynamic unknown network conditions under the presence of big-size obstacles. The proposed method ABNN designed to solve the problems of repeated hunting in the presence of big-size obstacles. The designing of multi-robot based hunting system is challenging as it has to address the various problems while performing the hunting. The targets or evaders can cope with some self-intelligence which means that their movements are entirely unknown and random. The evaders change their positions by sensing the nearby robots (which moving towards evaders to catch them). The problem of hunting presented in this paper is different from the regular multi-robot cooperative path planning problem, and foraging problem as the nature of evaders is dynamic and irregular. The fundamental contributions of this work are:

- We designed the simulation model of efficient cooperative hunting algorithm by multi-robots.

- We optimized the cooperative hunting algorithm concerning significant size obstacles and dynamic sensing capabilities for dynamic environments.

- The mechanism of implicit robots proposed to accurately guide the path to catch the dynamic inaccessible evaders by avoiding the big-sized obstacles.

- This method mainly based on properties in which pursing process can adaptively change, and movement of a robot can be guided under real time to not only pursue the target cooperatively but also faster as compared to previous work.

Paper organized as follow. In section II, the recent methods for multi-robot cooperative communications discussed. Section III presents the proposed problem definition, algorithm, architecture, and formulations. In section IV, different network conditions, their parameters, and performance metrics discussed for experimental analysis. In section $\mathrm{V}$, conclusion and future work discussed.

\section{RELATED WORK}

This section presents the brief review recent methods proposed on multi-robot based hunting problem.

Zhi-Qiang Cao et al. [21] initially reported the first approach to perform the cooperative hunting using multiple robots. They introduced Cooperative Local Interaction (CLI) method to achieve cooperative hunting. This approach was based on several assumptions and designed by considering the known environments. With this method, every robot finds its current state and then takes the decision based on invader and nearby robots information. However, this approach is practically not possible in real time environment where the robots and evaders are dynamic and frequently changing their positions. Our work is different from this work as we are working on the entirely adaptive environment.

Followed by CLI approach, in 2009 another method proposed for multi-robot hunting by Wenwen Zhang et al. [22]. They introduced the hunting method for multi-robot systems based on local interaction. This was the first approach reported in the unstructured and dynamic environment. Their process of hunting is composed of three phases such as initial leader-fixed following and search phase, leader changeable following and search phase, and finally the hunting phase. They designed the event-trigger communication method by utilizing the state of evader's observation. Our work is based on multirobot hunting for many dynamic evaders under the 
real-time environment by considering the small to substantial obstacles.

In [20], authors designed the bioinspired neural network based approach for real-time cooperative evader hunting using the team of multi-robots. To the best of our knowledge, this was the first approach considering the robots and evaders dynamics under the real-time environment with consideration of differently sized obstacles. This approach based on pursuing alliances of robots to catch the evaders effectively. The evaders hunting performed by designing the shunting equation. The working of artificial neural network (ANN) is based on shunting equation [35]. Our strategy based on this work only with consideration of solving the repeated hunting problem.

In [23], Zhiqiang Cao et al. proposed another local sensing based approach proposed for hunting. This approach designed for the multiple autonomous robots under the unstructured mode - free environments. Their method based on local sensing and effective sector. The task of hunting is design through the three different states such as search state, round obstacle state as well as hunting state. The cooperation among robots performed locally only. However, very importantly, they designed the evaders with escape strategy similar to [20]. Their approach addresses the unknown motion based evaders catching the problem. However, this approach is not considering the multi-robot based evaders catching; the catching of evader is performed by single robot only through local robots cooperation to reach the unknown evaders.

In [24], authors proposed the study on multi-robot cooperative hunting behavior through the mathematical model. They designed the mathematical model to represent the multi-robot based cooperative hunting problem. They presented the serious of equations to solve the problem of multi-robot based target hunting. This approach based works on waiting time required to catch the evaders.

In [25], recently Zongrui Huang et al. proposed bioinspired neural network based multi-AUV (Autonomous Underwater Vehicle) cooperative hunting method especially for the 3D underwater environment under the obstacle. Initially, the representation of AUV 3D system performed by the biological inspired neural network in which every neuron in the neural network is having the one-to-one correspondence with grid map position. Then the neurons activity value is utilized to direct the every hunting AUV navigation as well as obstacle prevention. Through this process, they efficient path was gathered for every hunting AUV to catch the target surrounded by all them. The author has not disclosed the dynamics of the target through their work.

In [26], the novel approach based on Lyapunov for cooperative hunting by multiple robots proposed. They first worked to obtain the relative dynamics from the robot-target relative kinematics. The extracted relative dynamics, as well as all robots dynamics, were used to derive the Lyapunov based local controller to stabilize individual robots in the system. They finally delivered the cooperative control rule through the addition of extra term in local controller. This represented mutual differentiations among the tracking errors.

In [27], the method for multi-robot hunting proposed by using the fuzzy interference approach. The hunting performed on virtual potential point. Authors utilized the kinematic constraints of wheeled robots; the motion trend was forecast by hunting robots as per the evaded motion location information. They used the fuzzy inference approach to control the multi-robot actions.

In [28], reported the robots group decision-based hunting approach for the multi-robot system. The method is very different and novel approach recently proposed. This method worked by considering the honeybee's behavior to hunt the new home if they lost the old home. Recently few works presented on multi-AUV hunting solutions in [29]-[31]. In our method, multi-robots used for evaders hunting cooperatively using the novel shunting model.

The proposed shunting model is design is based on previous shunting model reported in [20]. The existing methods studied in this paper suffered from problems like: (a) inefficient to handle the robotic dynamics, (b) current solutions are not adaptive enough to hunt the evaders as per the system environment and conditions, (c) the most of methods suffering from the problem of repeated hunting due to inaccessible sensing range or big-size obstacles, (d) this may lead to increase the number of steps required to catch, pursue and find the evaders. We redesigned the bioinspired neural network to hunt the many evaders to solve above-said problems.

\section{AdAPtive Multi Robot Co-Operative Hunting}

\section{A. Problem definition}

This paper presents the adaptive approach for multirobot based cooperative hunting. We first discuss the root problem of multi-robot hunting in this section. The system is assumed to be the group of collection robots, evaders, and obstacles. The robots don't know the environment as well as positions of evaders. However, robots are just aware of the total number evaders to catch. The robots are represented by team $\mathrm{T}$ which consists of the set of robots as rice, $\mathrm{I}=1,2, \ldots, \mathrm{n}$. The evaders is represented as e $\mathrm{j}, \mathrm{j}=1,2, \ldots, \mathrm{m}$. The robot nodes equipped with an omnidirectional antenna with the ability of $360^{\circ}$ visual capability as well as coordination with other robots. The cooperation among robots is performed by recognizing each other and then detecting the evaders. Additionally, the evaders are assumed to have the same processing capabilities as robots except for the cooperation and communication capabilities. However, evaders are build using self-intelligent manner so that can escape easily from catching whenever they sense any robots near to them as mentioned in [20].

There are research challenges associated with real-time cooperative hunting process such as:

\footnotetext{
- Performing the cooperative hunting efficiently
} 
under the presence of one or more evader.

- Hunting evaders are efficiently operating with less number of steps and burden.

- $\quad$ Detecting the evaders

- Detecting obstacles and preventing collisions

The above problems are addressed by [20] with the real-time cooperative hunting by multi-robots using the dynamic BNN. We are considering that the flags, hunting process, and other information are similar to [20] for this work. The real problem with existing dynamic BNN is the position information of evaders that cannot be utilized by BNN due to limited sensing radius of robots. Also, the complete environment is unknown to robots. This outputs resulted in the increasing number of steps for pursuing and finding to catch the evaders under the presence of different size obstacles. Due to significant sized barriers, the robots failed to detect the appropriate direction towards (as detection range of robots blocked by substantial issues) which the task of searching must perform. So this is the real problem associated with existing dynamic BNN.

\section{B. Proposed Solution}

To solve the problem of repeated hunting and to perform the hunting process adaptive and faster, we introduced the implicit robot concept in existing BNN. To present proposed concept, we first elaborate the BNN model for real-time cooperative hunting process, then introduce the implicit robot selection criteria.

\section{a. Hunting Process}

The algorithm for hunting based on some initial flags setting for both robots and evaders in a network:

$$
\text { Stat }\left(r^{i}\right)=\left\{\mathrm{S}^{\mathrm{r}} \mid \mathrm{S}^{\mathrm{r}} \in 0,1,2,3\right\}
$$

Where, status for each robot is among either of four states such as 0 (the robot broken), 1 (the robot is searching), 2 (the robot is walking, i.e., pursuing) and 3 (the robot is catching evader). Similarly, flags for evaders are changes dynamically as per their current state of action.

$$
\operatorname{Stat}\left(\mathrm{e}^{i}\right)=\left\{\mathrm{S}^{\mathrm{e}} \mid \mathrm{S}^{\mathrm{e}} \in 0,1,2\right\}
$$

Where, status for each evader is among either of three states like 0 (evader is still unknown), 1 (evader is known), and 2 (evader caught). Algorithm 1 is showing the proposed hunting -algorithm using adaptive BNN method.

\section{b. ABNN Model}

In this section, we presented the ABNN model in which robot considered as the main part. With dynamics of the robot, we modified the neural network to address the challenges associated with existing BNN model. The $\mathrm{BNN}$ model size set to maximum detection radius $\mathrm{R}$ of every robot. The distance from BNN model to neuron at a location of the robot represented via constraint:

$$
0<=\operatorname{dist}\left(P_{i}, P_{j}\right)<=R
$$

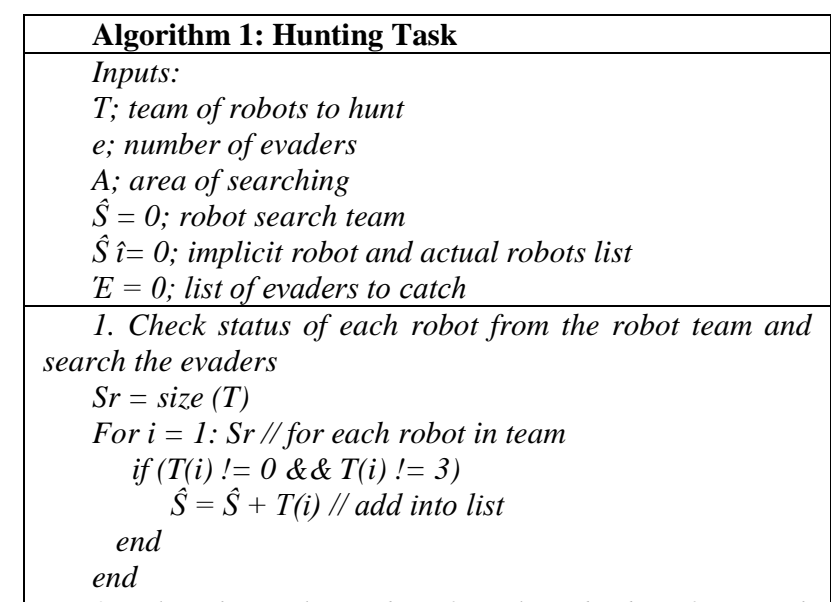

2. Select the implicit robots for selected robots for search evaders

$$
\begin{aligned}
& \mathrm{Sr}=\operatorname{size}(\hat{S}) \\
& \text { for } i=1: S r / / \text { for each selected robot in team } \\
& \hat{\imath}=\operatorname{algorithm} 2(\hat{S}(i)) \\
& \hat{S} \hat{\imath}=\hat{S} \hat{\imath}+\hat{\imath} / / \text { add into list } \\
& \text { end } \\
& \text { end }
\end{aligned}
$$

3. Sorting the selected list according ascending order $\hat{S} \hat{\imath}=\operatorname{sort}(\hat{S} \hat{i}$, 'asc')

4. Pursing State and catching state

for $i=1: S r / /$ for each selected robot in team

if $(\hat{S} \hat{i}(i)==2) / /$ if robot is in search state

$e_{o}=$ pursue ( $\hat{S} \hat{i}(i)$, e) // Robot pursuing current evader marked as old evader if $\left(\operatorname{dist}\left(\hat{S} \hat{\imath}(i), e_{o}\right)-\operatorname{dist}\left(\hat{S} \hat{\imath}(i), e_{n}\right)<=0 / /\right.$ found new

evader

$\hat{S} \hat{i}=\hat{S} \hat{i}-\hat{S} \hat{i}(i) / /$ remove current robot from the list

$$
\begin{aligned}
& \text { end } \\
& \text { end } \\
& \text { end }
\end{aligned}
$$

5. Search and Pursing State

for $i=1: S r / /$ for each selected robot in team if $(\hat{S} \hat{\imath}(i)==1) / /$ if robot is free to search evader // then assign that robot to the task of pursing assign ( $\left.\hat{S}_{\hat{i}}(i), \quad E(j)\right)$ // assigning current robot to catch evader $e_{j}$ and add into the evaders list

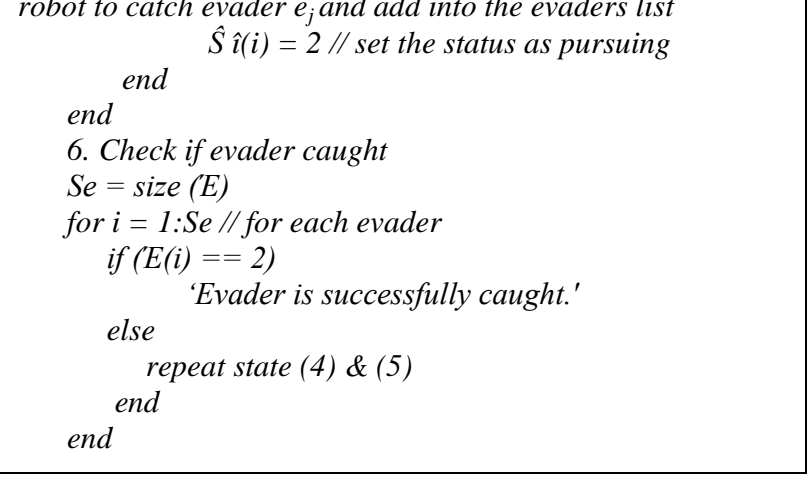

Where the distance between two neurons or position is computed such as:

$$
\operatorname{dist}\left(P_{i}, P_{j}\right)=\sqrt{\left(x_{i}-x_{j}\right)^{2}+\left(y_{i}-y_{j}\right)^{2}}
$$

where $\left(\mathrm{x}_{\mathrm{i}}, \mathrm{y}_{\mathrm{i}}\right)$ and $\left(\mathrm{x}_{\mathrm{j}}, \mathrm{y}_{\mathrm{j}}\right)$ is the information of $i^{\text {th }}$ and the 
$j^{\text {th }}$ cell locations in grid system respectively.

\section{c. Addressing repeated hunting problem}

Toduces a novel concept of using an implicit robot. The implicit robot is mainly because; robot should be guided quickly to catch the moving evaders by avoiding the significant obstacles automatically. As there is the team of robots, each robot equipped with the functionality of implicit o solve the problem of repeated hunting the paper intrrobot. So any robot with an implicit robot may lead fast discovery of evader and can inform the other robots. Use of implicit robot may be reliable if the network is more dynamic and complex.

Therefore, we are modifying the existing BNN model to perform hunting task more efficiently and adaptively in the presence of significant sized obstacles. The modification mainly based on the use of implicit robot functionality in dynamic BNN to catch the evaders. The selection of implicit robot is performed based on the following rules: (these rules are contradicting if we have the virtual robot as an implicit robot)

- At the time of network deployment, implicit robot $\left(r^{i}\right)$ should deploy over the boundary of BNN.

- The $r^{i}$ should be accessible by robot nodes and should help other robots to move close to evaders

- There should not be any obstacle between $r^{i}$ and the actual robot

In general, the selection of implicit robot is performed by:

$$
P^{r i}=\left\{\operatorname{dist}\left(P^{n}, P^{e}\right) \leq \operatorname{dist}\left(P^{k}, P^{e}\right), P^{k} \in G N\right\}
$$

Where $P^{r i}$ and $P^{e}$ represent a cell location of the implicit robot \& evader respectively, $P^{n}$ represents the nearest cell to an evader and $P^{k}$ represents any cell of the grid network. GN denotes the 2-D grid network which contains the information of cell allocations. The 0 value in any cell location means that cell is free and one means obstacle occupies it. The equation (5) extracts the position for implicit robot towards the evader to catch for the particular actual robot. Algorithm 2 below presents the process of selecting the implicit robot.

\begin{tabular}{|l|}
\hline Algorithm 2: Implicit Robot Selection \\
\hline Input: \\
$G N ; / / 2 \mathrm{D} \mathrm{BNN}$ based grid cell network \\
$r^{n} ; / /$ number of catching robots \\
$e^{m} ; / /$ number of evaders \\
$R ; / /$ detection range of robot \\
Output: \\
$P^{r i} ; / /$ position selected for implicit robot \\
1. Extract initial position of each robot \\
for $\mathrm{i}=1: \mathrm{n}$ \\
$\mathrm{P}^{\mathrm{i}}=\left[\mathrm{x}^{\mathrm{i}}, \mathrm{yr}^{\mathrm{i}}\right]$ \\
end \\
2. Extract initial area of each evader \\
for $\mathrm{j}=1: \mathrm{m}$ \\
$\mathrm{Pe}^{\mathrm{j}}=\left[\mathrm{xr}^{\mathrm{j}}, \mathrm{yr}^{\mathrm{j}}\right]$ \\
End \\
3. Compute distance each robot and evader pair \\
\hline
\end{tabular}

Copyright @ 2018 MECS

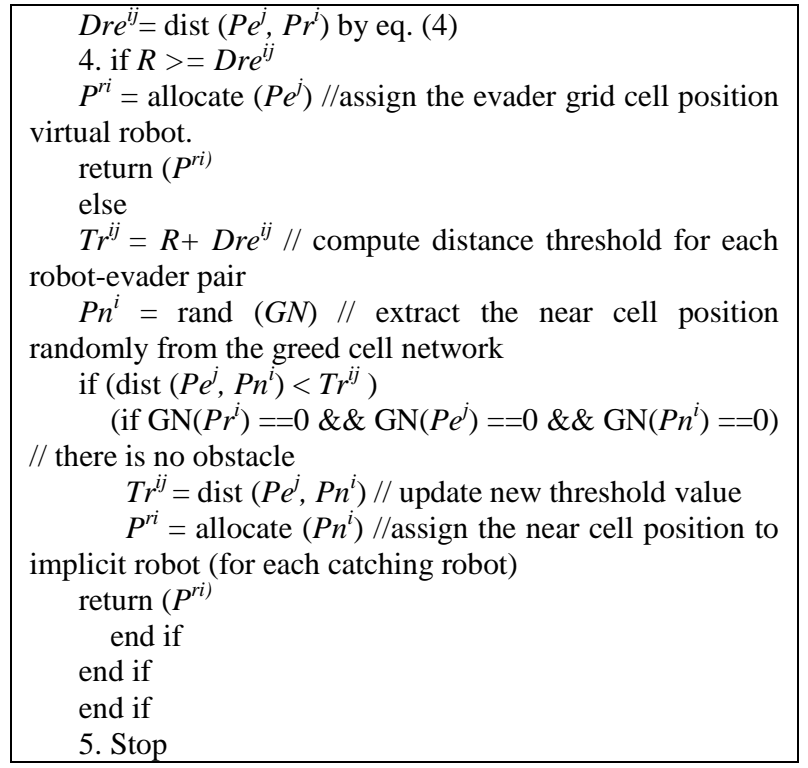

\section{d. Proposed Shunting Equation}

As stated in [20], the motions of robots fall under the similar alliance are used to perform the real-time cooperative hunting by considering the dynamic activities of the neural network. The space of environment represented in 2-D matrix GN to each robot in team T. The path planning from the current robot location to the selected implicit robot position done by the existing BNN shunting equation represented by:

$$
\begin{gathered}
\frac{d x_{i}}{d t}=-A x_{i}-\left(D+x_{i}\right)\left[I_{i}\right]^{o-}+\left(B-x_{i}\right)\left(\left[I_{i}\right]^{I R+}+\right. \\
\left.\sum_{j=1}^{k} \omega_{i j}\left[x_{j}\right]^{+}\right)
\end{gathered}
$$

Where,

${ }_{X i}$ is the neural activity of the $i$ th neuron; $A$ representing the passive rate; $B$ represents the upper bound and D represents the lower bound. $K$ is the number of neural connections of the $i$ th neuron to its neighboring neurons within the receptive field. The $w_{i j}$ is the lateral connection weight from the $i$ th neuron to the $j$ th neuron, which is a function of the distance; and $\left[I_{i}\right]^{\mathrm{IR}}$ and $\left[I_{i}\right]^{\mathrm{o}}$ is the excitatory and inhibitory inputs to the current neuron. Where the implicit robot and the surrounding positive neurons are the excitatory input, and the obstacles mean the inhibitory input. Function $[x]^{+}$is a linear-abovethreshold function defined as $[x]^{+}=\max \{x, 0\}$, and $[x]$ is defined as $[x]^{-}=\max \{-x, 0\}$.

As the environment is dynamic, the evader globally sensing the whole state space of the BNN and the obstacle always keeps its corresponding neuron activity at a shallow level. So the actual robot selects the nearby neuron with tough next position towards implicit robot using:

$$
P_{n}=\max \left\{x_{i}, i=1,2, \ldots, m\right\},
$$

Where $x_{i}$ is the activity of all the neighboring neurons; $\mathrm{Pn}$ is the position of the next neuron which is having the maximum activation of all other neurons.

However, its required that the number of steps to 
perform path estimation from actual robot to implicit robot and robot to evader leads to computation overhead in the network. Therefore to minimize the computation overhead, we modified the existing shunting equation as:

$$
\begin{gathered}
\frac{d_{x i}}{d t}=-A_{x i}-\left(D+x_{i}\right)\left[I_{i}\right]^{o-}+\left(B-x_{i}\right)\left(\left(\left[I_{i}\right]^{e+}+\right.\right. \\
\left.\left.\sum_{j}^{k} \omega_{i j}\left[x_{j}\right]^{+}+E S_{i}\right)\right)
\end{gathered}
$$

Where the $E S_{i}$ represents implicit robot approach which senses evaders quickly, as early they can. The function $E S_{i}$ defined as:

$$
E S_{i}=\left(\operatorname{rand}(1) / \eta_{i}\right) \Delta_{i}
$$

Where, rand (1) generated any positive number in between 0.1 to 0.99 and $\eta_{i}$ is distance computed between actual robots $P r^{i}$ to implicit robot $P^{r i}$ using equation (4). Finally, $\Delta_{i}$ is an important factor; this decides the moving direction of the particular robot from their current location to the next location. This is the difference between two lines represented by the angle. It is computed by:

$$
\Delta_{i}=\operatorname{atan} 2 d\left(C\left(\operatorname{Pr}^{i}, P^{r i}\right), D\left(\operatorname{Pr}^{i}, P^{r i}\right)\right)
$$

The function $\operatorname{atan} 2 d$ returns the four-quadrant inverse tangent angle in degrees to select the moving direction. Inside this function, $\mathrm{C}$ is cross product function and $\mathrm{D}$ is dot product function to select the optimum angles. These two functions return the scalar products of two locations. To best of our knowledge, this is the first time we are using the angle computation for the real-time 2D environment of hunting problem.

With this proposed model, the number of steps required to catch the evaders may be more, but the steps needed to pursue, step to find and overall hunting process time minimized.

\section{PERFORMANCE EVALUATION}

The experimental analysis of proposed work is performed using the MATLAB simulator. We investigated the performance of proposed method compared to the 4 state-of-art multi-robot hunting methods [24]-[27]. We designed and evaluated both existing hunting models and proposed ABNN model. To claim the effectiveness of the proposed approach, in this paper we designed different size grid systems small (10x10) to large $(30 \times 30)$ with large U shape obstacle and multiple robots and evaders. The simulation parameters for hunting task as well as neural network model (NNM) presented in table 1 below:
Table 1. Simulation parameters

\begin{tabular}{|c|c|c|}
\hline Parameter & Value & Note \\
\hline$R s$ & 2 & Onboard sensor radius (m)-Hunting \\
\hline $\mathrm{Rc}$ & 1 & $\begin{array}{c}\text { Formatting circle radius (m)- } \\
\text { Hunting }\end{array}$ \\
\hline $\mathrm{Nc}$ & 4 & $\begin{array}{c}\text { Number of robots to catch one } \\
\text { evader-Hunting }\end{array}$ \\
\hline $\mathrm{Ve}$ & 0.3 & Evader velocity (m/s)-Hunting \\
\hline $\mathrm{Vr}$ & 0.5 & Robot velocity (m/s)-Hunting \\
\hline $\mathrm{A}$ & 25 & Passive decay rate-NNM \\
\hline $\mathrm{B}$ & 1 & Upper bound-NNM \\
\hline $\mathrm{D}$ & 1 & Lower bound-NNM \\
\hline $\mathrm{E}$ & 100 & Large +ve Constance-NNM \\
\hline $\mathrm{Re}$ & 10 & Evader effective radius (m)-NNM \\
\hline $\mathrm{Ro}$ & 1 & Obstacle effective radius (m)-NNM \\
\hline $\mathrm{R}$ & 10 & Robot detection rate-Hunting \\
\hline
\end{tabular}

As the proposed model based on the method reported in [20], first we demonstrated the comparative results between proposed method and BNN [20] method. Figure 1,2 and 3 explaining processing to catch the moving evaders by multi-robots. Figure 1 is showing the initial positions for two robots and one evader with two obstacles. Figure 2 show that both robots start their searching and catching process using proposed method towards evader. Due to the intelligence of evader, evader moves their current position to beat the robots as shown in figure 3. As proposed approach designed with capabilities of addressing the dynamics of evaders, the robots finally estimated the trajectories to catch evaders efficiently showing in figure 3 .

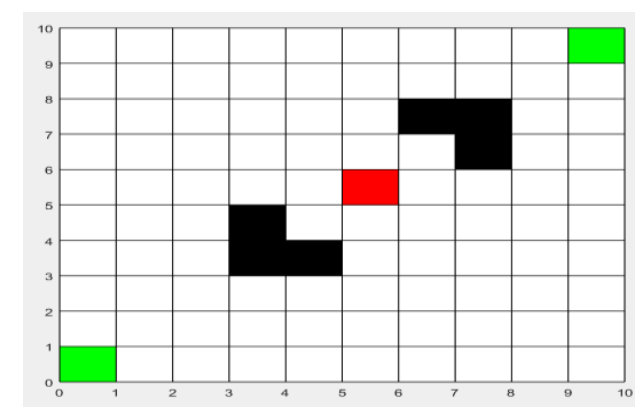

Fig.1. Initial position of robots and evader

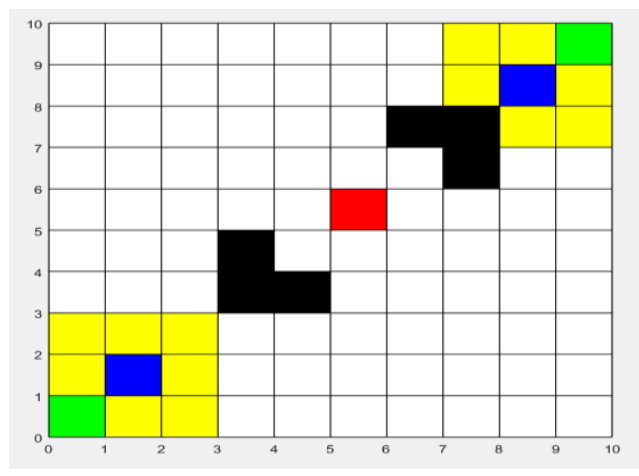

Fig.2. Start hunting process 


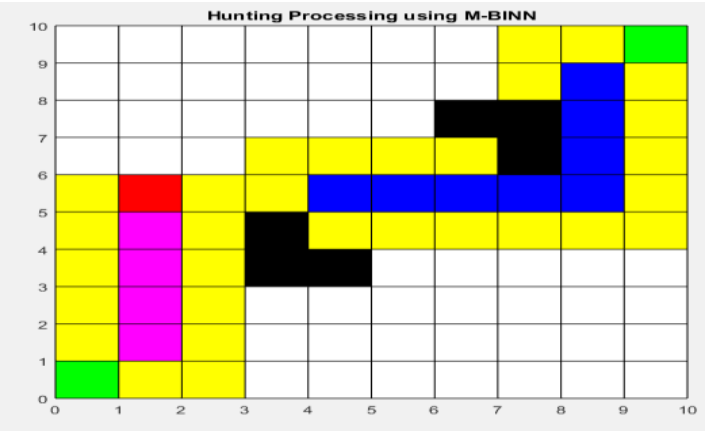

Fig.3. Final hunting of evader by multi-robots

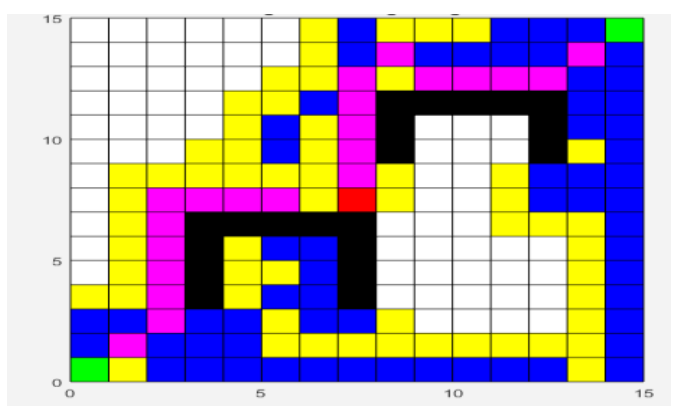

Fig.4. Evader catching by two robots in significant size obstacles using existing BNN model

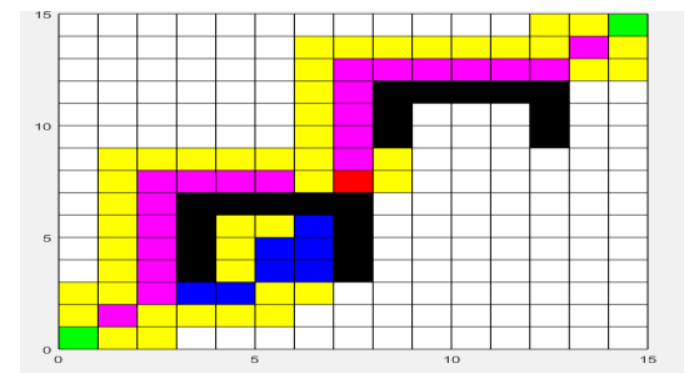

Fig.5. Evader catching by two robots in significant size obstacles using ABNN Mode

Figure 4 and 5 are demonstrating the difference between existing BNN model and proposed ABNN for hunting the evaders in a dynamic environment. The yellow grid cells were indicating the steps of pursuing required catching the evader. The blue grid cells were indicating the search directions needed to find the evader. The magenta grid cells are indicating the actual steps necessary to detect evader. The black cell represents the obstacles. The pursuing steps and search steps required to catch evaders in the existing case is primarily due to significant sized barriers. The significant sized issues lead to the problem of repeated hunting in the current method. However, in the same scenario, the steps are minimized to a great extent due to the use of implicit robots to guide the faster direction to catch the evaders by adaptively avoiding the evaders. The comparative study of the existing and proposed method elaborated through four key performance metrics such as steps to catch, steps to pursue, steps to find and hunting process time. Below graphs for each network size showing the performance analysis between existing $\mathrm{BNN}$ and proposed ABNN models.

Figure 6 is showing the total number of steps required to catch the evader for different size of networks such as $10 \times 10,15 \times 15,20 \times 20,25 \times 25,30 \times 30$ using BNN and ABNN methods. The systems are designed using two robots and one evader scenario with the presence of two prominent sizes (U) shaped obstacles. There is not much improvement observed in steps reduction to catch the evader for each size network for the proposed method as compared to the existing technique. However, figure 4 and 5 are showing the effectiveness and efficiency of proposed $\mathrm{ABNN}$ method.

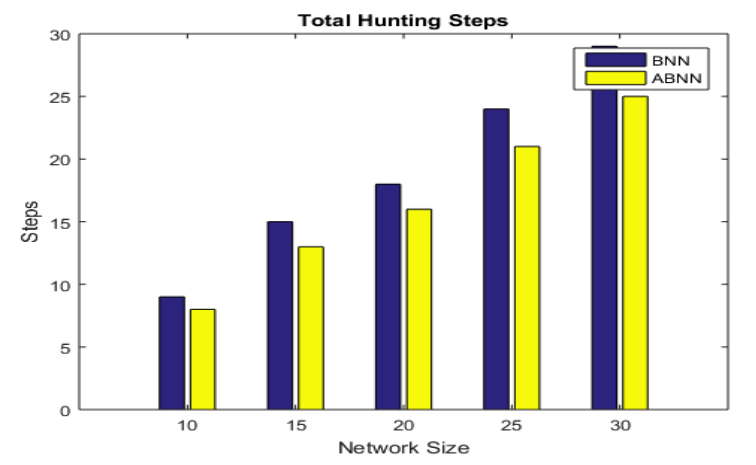

Fig.6. Performance analysis of number of steps required to catch evader

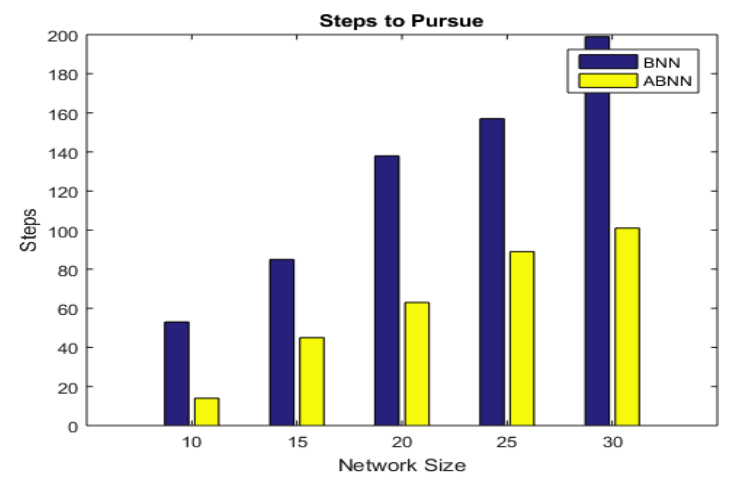

Fig.7. Performance analysis of number of pursue required to catch evader

As for showing in figure 6 and 7 , the problem of repeated hunting is solved by $\mathrm{ABNN}$ technique at the great level. The proposed method reduced the total number of steps to peruse the evader by almost three times lesser than BNN model for each size of the network. This is due to using of an implicit robot which helps to guide the direction of moving evader accurately to reduce the extra burden of search and pursuing evaders repeatedly.

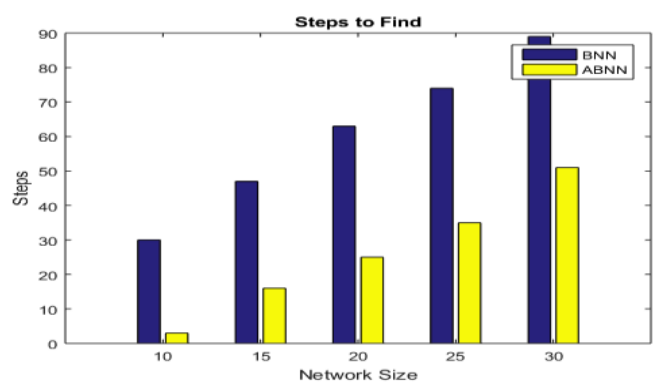

Fig.8. Performance analysis of the number of finds required to catch evader 


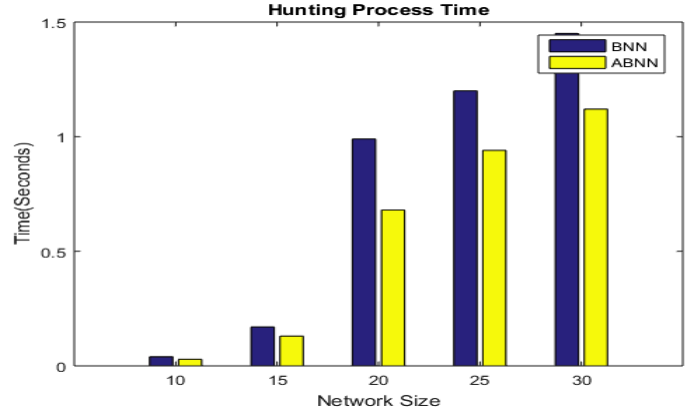

Fig.9. Total hunting time analysis

Finally, figure 9 claim the time reduction in the process of hunting the evader. If their less number of steps required pursuing and finding the evaders, then total hunting this also reduced. The proposed ABNN model outperformed the existing BNN model for each network size scenarios under the selected circumstance.

Table 2 presents the average performance of ABNN method as compared to other recent hunting methods in presence of big obstacles.

Table 2. Performance evaluation with similar methods

\begin{tabular}{|c|c|c|c|c|}
\hline Methods & $\begin{array}{c}\text { Steps to } \\
\text { Hunt }\end{array}$ & $\begin{array}{c}\text { Steps to } \\
\text { Pursue }\end{array}$ & $\begin{array}{c}\text { Steps to } \\
\text { Search }\end{array}$ & $\begin{array}{c}\text { Hunting } \\
\text { Time }\end{array}$ \\
\hline$[24]$ & 18.3 & 120 & 58 & 0.82 \\
\hline$[25]$ & 19.1 & 140 & 67 & 0.99 \\
\hline$[26]$ & 16.2 & 93 & 54 & 0.78 \\
\hline$[27]$ & 20.3 & 88 & 50 & 0.73 \\
\hline$[20]$ & 19.4 & 128 & 59.6 & 0.84 \\
\hline Proposed & $\mathbf{1 5 . 4}$ & $\mathbf{5 2}$ & $\mathbf{2 0 . 8}$ & $\mathbf{0 . 6 8}$ \\
\hline
\end{tabular}

As observed in table 2, the proposed method shows the significant improvement in performance as compared to other state-of-art methods by using the proposed dynamic cooperating multi-robot hunting method. The ABNN method not only minimizes the total efforts required to hunt evaders under the presence of big sized obstacles, but also reduce the total hunting time due to multidirectional capability of implicit robot which virtually captures the right directions towards the evaders. The state-of-art methods, does not have the provision to handle the big sized obstacles in network while hunting the evaders, hence this increases the overall efforts required to hunt actual evaders.

\section{CONCLUSION AND FUTURE WORK}

In networks like underwater vehicle network, robotic dynamics, etc. the concept of multi-robot cooperation is widely used to solve the problems like task allocation, evaders hunting, target select, etc. In this paper, we presented the method for cooperative hunting using the multi-robots system for high level of system dynamics. We proposed the adaptive BNN model based on existing BNN model. We modified the current BNN model to solve the problems of repeated pursuing and searching tasks to catch the many evaders due to the presence of significant size obstacles and limited sensing range of robots. This can be done by modifying the shunting equation to optimize the evader catching performance via neurons updating function. The simulation results for different types of networks claim effectiveness and efficiency of proposed work regarding computational efforts and steps required as compared to recent methods. For future work, it will be interesting to use the optimization techniques to further optimize the performance of current BNN model to select the next neuron function.

\section{REFERENCES}

[1] J J.-C. Latombe, "Robot motion planning," Kluwer Academic Publishers, 1991.

[2] M. Erdmann, T. Lozano-Pérez, "On multiple moving objects," IEEE Int. Conf. on Robotics and Automation, 1986.

[3] P. Svestka, M.H. Overmars, "Coordinated path planning for multiple robots," Robotics and Autonomous Systems, 23 (1998).

[4] S. Leroy, J.P. Laumond, T. Siméon, "Multiple path coordination for mobile robots: a geometric algorithm," Int. Joint Conf. on Artificial Intelligence, 1999.

[5] C. W. Warren, "Multiple robot path coordination using artificial potential fields," IEEE Int. Conf. on Robotics and Automation, 1990.

[6] K. Azarm, G. Schmidt, "Conflict-free motion of multiple mobile robots based on decentralized motion planning and negotiation," IEEE Int. Conf. on Robotics and Automation, Albuquerque, New Mexico, 1997.

[7] M. Bennewitz, W. Burgard, S. Thrun, "Optimizing schedules for prioritized path planning of multi-robot systems," IEEE Int. Conf. on Robotics and Automation, Seoul, Korea, 2001

[8] M.C. Clark, S.M. Rock, J.-C. Latombe, "Motion planning for multiple mobile robot systems using dynamic networks," IEEE Int. Conf. on Robotics and Automation, Taipei, Taiwan, 2003.

[9] Ralf Regele, Paul Levi, "Cooperative Multi-Robot Path Planning by Heuristic Priority Adjustment," Proceedings of the 2006 IEEE/RSJ International Conference on Intelligent Robots and Systems October 9 - 15, 2006, Beijing, China

[10] R. K. Sharma and D. Ghose, "Collision avoidance between UAV clusters using swarm intelligence techniques,” Int. J. Syst. Sci., vol. 40, no. 5, pp. 521-538, May 2009.

[11] S. Sariel, T. Balch, and N. Erdogan, "Naval mine countermeasure missions," IEEE Robot. Autom. Mag., vol. 15, no. 1, pp. 45-52, Mar. 2008.

[12] Z. Cao, M. Tan, L. Li, N. Gu, and S. Wang, "Cooperative hunting by distributed mobile robots based on local interaction,” IEEE Trans. Robot., vol. 22, no. 2, pp. 403407, Apr. 2006.

[13] K. Tanaka and E. Kondo, "A scalable localization algorithm for high dimensional features and multi-robot systems," in Proc. IEEE Int. Conf. Network., Sens. Control, Sanya, China, Apr. 2008, pp. 920-925.

[14] S. K. Chalup, C. L. Murch, and M. J. Quinlan, "Machine learning with AIBO robots in the four-legged league of RoboCup," IEEE Trans. Syst., Man, Cybern., Part C: Appl. Rev., vol. 37, no. 3, pp. 297-310, May 2007.

[15] W. Sun, L. Dou, H. Fang, and H. Zhang, "Task allocation for multi-robot cooperative hunting behavior based on improved auction algorithm," in Proc. Chin. Control Conf., 
Kunming, China, Jul. 2008, pp. 435-440.

[16] D. J. Pack, P. DeLima, G. J. Toussaint, and G. York, "Cooperative control of UAVs for localization of intermittently emitting mobile targets," IEEE Trans. Syst., Man, Cybern., Part B: Cybern., vol. 39, no. 4, pp. 959970, Aug. 2009.

[17] M. Xu, Z. Pan, H. Lu, Y. Ye, P. Lv, and A. E. Rhalibi, "Moving target pursuit algorithm using improved tracking strategy," IEEE Trans. Comput. Intell. AI Games, vol. 2, no. 1, pp. 27-39, Mar. 2010.

[18] H. Zhang, Y. Wu, and Y. Cen, "Multirobot dynamic pursuit scheme based on behavior-merging and task decision-making technology," in Proc. World Congr. Comput. Sci. Inf. Eng., Los Angeles, CA, Mar.- Apr. 2009, pp. 575-580.

[19] J. T. Feddema, C. Lewis, and D. A. Schoenwald, "Decentralized control of cooperative robotic vehicles: Theory and application," IEEE Trans. Robot. Autom., vol. 18 , no. 5, pp. 852-864, Oct. 2002.

[20] Jianjun Ni, Member, IEEE, and Simon X. Yang, "Bioinspired Neural Network for Real-Time Cooperative Hunting by Multi-robots in Unknown Environments," IEEE TRANSACTIONS ON NEURAL NETWORKS, VOL. 22, NO. 12, DECEMBER 2011

[21] Z. Q. Cao, M. Tan, S. Nahavandi \& N. Gu, "Cooperative Hunting by Multiple Mobile Robots Based on Local Interaction", Cutting Edge Robotics, ISBN 3-86611-038-3, pp. 784, ARS/plV, Germany, July 2005

[22] W. Zhang1, J. Wang2, Z. Cao1, Y. Yuan1, and C. Zhou1, "A Local Interaction Based Multi-robot Hunting Approach with Sensing and Modest Communication", Springer-Verlag Berlin Heidelberg 2009

[23] Z. Cao1,*, C. Zhou1, Lo. Cheng1, Y. Yang2, W. Zhang1 and M. Tan1, "A Distributed Hunting Approach for Multiple Autonomous Robots", International Journal of Advanced Robotic Systems, 2012

[24] Y. Song,1,2 Y. Li,1 C. Li,3 and X. Ma1, "Mathematical Modeling and Analysis of Multirobot Cooperative Hunting Behaviors", Hindawi Publishing Corporation, Journal of Robotics, Volume 2015.

[25] Z. Huang, D. Zhu, B. Sun, "A multi-AUV cooperative hunting method in 3-D underwater environment with obstacle", Engineering Applications of Artificial Intelligence, Volume 50, April 2016.

[26] H. Shen; N. Li; S. Rojas; L. Zhang, "Multi-Robot Cooperative Hunting", 2016 International Conference on Collaboration Technologies and Systems (CTS)

[27] Y. Duan; X. Huang; X. Yu, "Multi-robot dynamic virtual potential point hunting strategy based on FIS", 2016 IEEE Chinese Guidance, Navigation and Control Conference (CGNCC)

[28] S. Momen; T. I. Lima; R. Siddika, "Group decision by house-hunting agents in multi-robot systems", 2016 2nd International Symposium on Agent, Multi-Agent Systems and Robotics (ISAMSR)

[29] Daqi Zhu1, Ruofan Lv1, Xiang Cao1 and Simon X. Yang, "Multi-AUV Hunting Algorithm Based on Bio-inspired Neural Network in Unknown Environments", International Journal of Advanced Robotic Systems, 2015.

[30] Jianjun Ni, Liu Yang, Liuying Wu, Xinnan Fan, "An Improved Spinal Neural System-Based Approach for Heterogeneous AUVs Cooperative Hunting", International Journal of Fuzzy Systems, February 2018, Volume 20, Issue 2, pp 672-686.

[31] Mingzhi Chen ; Daqi Zhu, "A Novel Cooperative Hunting Algorithm for Inhomogeneous Multiple Autonomous Underwater Vehicles", IEEE Access ( Volume: 6 ), 2018.
[32] Krishna S.R.M., Seeta Ramanath M.N., Kamakshi Prasad V, "Optimal Reliable Routing Path Selection in MANET through Novel Approach in GA", I.J. Intelligent Systems and Applications, 2017, 2, 35-41.

[33] Seema Verma, Prachi, "Communication Architecture for Underwater Wireless Sensor Network", I.J. Intelligent Systems and Applications, 2015, 6, 67-74

[34] Md. Ashraf Uddin, Md. Mamun, Md.Rashid, Md. MustafizurRahman, "Priority Metric based Ad Hoc Routing for Underwater Sensor Network", ", I.J. Intelligent Systems and Applications, 2013, 12, 1-11.

[35] Huiyan Kang, Ligeng Si, "Stabilitty of Anti-periodic Solutions for Certain Shunting Inhibitory Cellular Neural Networks", I.J. Intelligent Systems and Applications, 2011, 5, 26-32.

\section{Authors' Profiles}

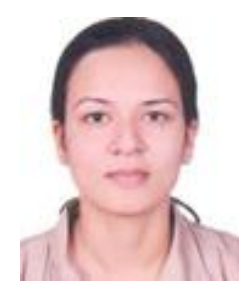

Poorva Agrawal was born in Pipariya, Madhya Pradesh State, India in 1987. She received her B.E degree in Computer Science from Rajiv Gandhi University, Bhopal in the year 2009, Masters in Computer Science Engineering from SGSITS, Indore in 2012 and currently pursuing her $\mathrm{PhD}$ in Computer Science from Symbiosis International University.

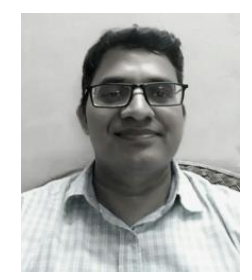

Himanshu Agrawal received Bachelor of Engineering in Electronics in 1994, Master of Technology in Computer Science in 2003 from Devi Ahilya University India and $\mathrm{PhD}$, in Electrical and Computer Engineering, RMIT University, Melbourne, Victoria, Australia in 2010.

During 1995-1998, he had served as an Instrumentation Engineer in India. Since 1999, he is working as an academician. During past 16 years, he served in academics holding different positions in India and Australia. Currently associated with Symbiosis International University as an Associate Professor since August 2011, he had authored papers in peer-reviewed journals and International conferences. His research interest includes Internet routing, Internet of Things, intrusion detection and security issues in Smart grid and future Internet design issues. Dr Agrawal is a regular reviewer of Journal of Network and Computer Applications.

How to cite this paper: Poorva Agrawal, Himanshu Agrawal, "Adaptive Algorithm Design for Cooperative Hunting in MultiRobots", International Journal of Intelligent Systems and Applications(IJISA), Vol.10, No.12, pp.47-55, 2018. DOI: 10.5815/ijisa.2018.12.05 